\title{
Enhanced Conductivity Along Lateral Homojunction Interfaces of Atomically Thin Semiconductors
}

\author{
Ying Jia, Teodor K. Stanev, Erik J. Lenferink and Nathaniel P. Stern* \\ Department of Physics and Astronomy, \\ Northwestern University, Evanston, Illinois 60208 USA
}

\begin{abstract}
Energy band realignment at the interfaces between materials in heterostructures can give rise to unique electronic characteristics and non-trivial low-dimensional charge states. In a homojunction of monolayer and multilayer $\mathrm{MoS}_{2}$, the thicknessdependent band structure implies the possibility of band realignment and a new interface charge state with properties distinct from the isolated layers. In this report, we probe the interface charge state using scanning photocurrent microscopy and gate-dependent transport with source-drain bias applied along the interface. Enhanced photoresponse observed at the interface is attributed to band bending. The effective conductivity of a material with a monolayer-multilayer interface of $\mathrm{MoS}_{2}$ is demonstrated to be higher than that of independent monolayers or multilayers of $\mathrm{MoS}_{2}$. A classic heterostructure model is constructed to interpret the electrical properties at the interface. Our work reveals that the band engineering at the transition metal dichalcogenides monolayer/multilayer interfaces can enhance the longitudinal conductance and field-effect mobility of the composite monolayer and multilayer devices.
\end{abstract}

\section{Introduction}

Transition metal dichalcogenides (TMDCs) are layered crystals with thickness-dependent band gaps, $1.29 \mathrm{eV}$ in a multilayer (ML) and $1.88 \mathrm{eV}$ for a monolayer (1L) of $\mathrm{MoS}_{2}$ [1] for example. The distinct electronic structure implies an energy band discontinuity at the interface in 1L and $\mathrm{ML} \mathrm{MoS}_{2}$. Recent Kelvin probe force microscopy has detected different electron affinities in 1L and $\mathrm{ML} \mathrm{MoS}_{2}$ and the conduction band offset at the boundary between two regions of the same region material but with different layer numbers [2] (which we refer to here as a $1 \mathrm{~L} / \mathrm{ML}$ homojunction). According to the electron affinity model, band realignment should occur near the interface and may create unique interfacial electronic states with properties distinct from those of the parent compounds 3. Examples of such emergent interfacial properties in heterostructures are high-mobility two-dimensional electron gases in $\mathrm{Al}_{x} \mathrm{Ga}_{1-x} \mathrm{As} / \mathrm{GaAs}$ [4], and interface superconductivity in $\mathrm{Bi}_{2} \mathrm{Te}_{3} / \mathrm{FeTe}$ [5]. Distinct from the "conventional semiconductor heterojunctions, experimental and calculated results have suggested localized metallic states at the $1 \mathrm{D}$ edges of $1 \mathrm{~L}$ and few-layer $\mathrm{MoS}_{2}$ [6 11, 14]. In a lateral 1L/ML $\mathrm{MoS}_{2}$ homojunction (Fig. 1a), such an edge state of ML MoS ${ }_{2}$ could

\footnotetext{
* n-stern@northwestern.edu
} 
influence the band realignment at the interface, complicating the electronic structure and the properties of the interface electrons.

Recent studies on TMDC 1L/ML homojunctions were primarily focused on photocurrent generation at the interfaces with source-drain contacts on opposite sides of the 1L/ML junctions [2, 12, 13]. Band realignment has been confirmed though the details are still under debate [13]. Current versus voltage $(I V)$ measurements were also performed with currents applied across the junction [12, 13] with non-linear $I-V$ curves consistently observed. The existence of conducting interfacial edge charge states at multilayer junctions separating distinct TMDC thicknesses has been measured [14], but the consequences of layer-sensitive composite structures for low-dimensional interfacial transport at the monolayer limit have not yet been investigated. In particular, the longitudinal transport properties of $1 \mathrm{~L} / \mathrm{ML}$ interfaces remain unstudied.

Here, we fabricate lateral $\mathrm{MoS}_{2} 1 \mathrm{~L} / \mathrm{ML}$ homojunctions and investigate the longitudinal transport properties of the interface charge state with source-drain bias applied along the interface. Interfacial band bending is confirmed by enhanced photoresponse along the junction boundary using scanning photocurrent microscopy. Electric conductivity of the $1 \mathrm{~L}$ and ML composite devices is measured and compared with that of the independent $1 \mathrm{~L}$ and ML $\mathrm{MoS}_{2}$ of the same flake. The higher effective conductivity of the composite devices indicates a notable contribution from the interface charge state. Our results outline an experimental approach to studying interfacial conduction properties of electronic states at layered TMDC homojunctions.

\section{Methods}

The $\mathrm{MoS}_{2}$ flakes were mechanically exfoliated from undoped crystals and deposited on heavily doped silicon substrates covered with 285-nm-thick $\mathrm{SiO}_{2}$ using elastic-film-assisted micro-mechanical exfoliation [16]. $\mathrm{MoS}_{2}$ flakes with both $1 \mathrm{~L}$ and ML regions were identified using optical microscopy and then confirmed using atomic force microscopy. Fig. 1b shows a typical as-exfoliated sample. This sample consists of 8 layers on one side and 1 layer on the other side, as shown in the inset of Fig. 1b. The 1L/ML structures were patterned into individual pieces with $\mathrm{Au}$ electrodes using e-beam lithography and $\mathrm{SF}_{6}$ reactive ion etching [17]. Each of the $1 \mathrm{~L}, \mathrm{ML}$, and $1 \mathrm{~L}+\mathrm{ML}$ devices were independently measured with a 4-point method. All devices were operated in a back-gating configuration to maintain the direct accessibility of an excitation laser with the heavily doped Si substrates acting as the back gates.

\section{Results}

Scanning photocurrent microscopy was used initially to probe the generation of carriers and band bending at the 1L/ML homo-interface. The measurement was performed in high vacuum (less than $1 \mathrm{mTorr}$ ) and at room temperature with laser excitation power of 70 $-80 \mu \mathrm{W}$ at several wavelengths as noted. In few-layer $\mathrm{MoS}_{2}$, the primary photocurrent mechanisms involve two processes, exciton generation by a photon with energy higher than optical band gap and free carrier creation by electric-field-assisted dissociation [18]. At the 


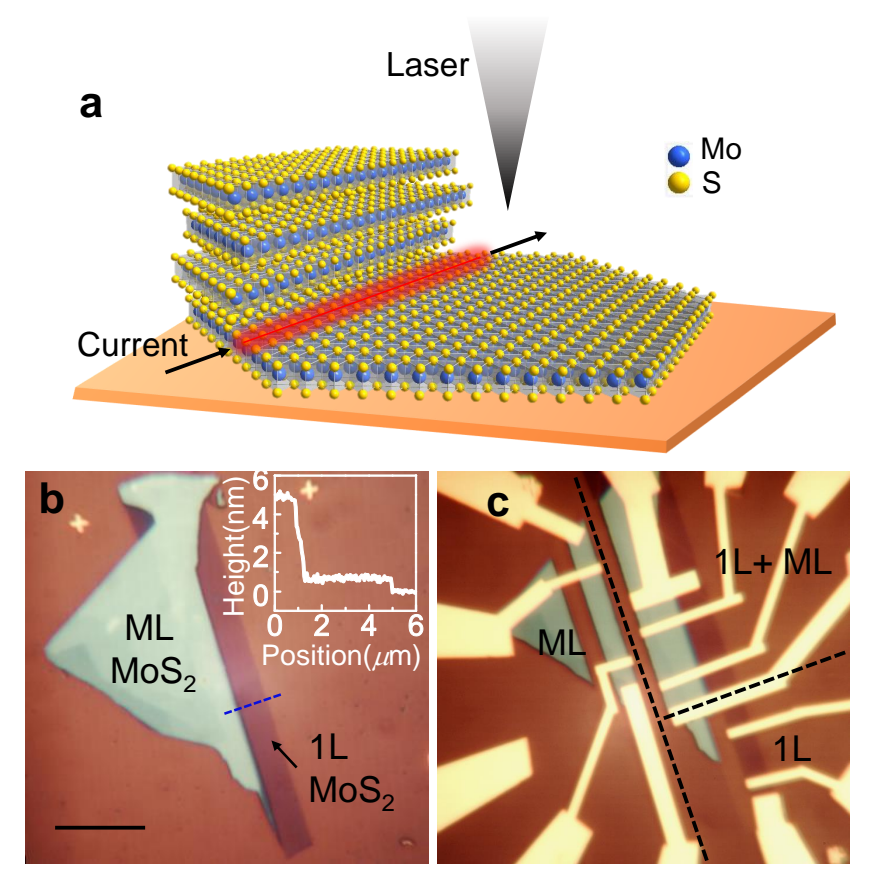

FIG. 1. Schematic and optical image of a $\mathrm{MoS}_{2}$ homojunction. (a) Schematic illustration of the 1L/ML homojunction and the experiment geometry. (b) Optical image of an as-exfoliated 1L/ML flake. The inset is the thickness profile along the blue dotted line. The scale bar is $10 \mu \mathrm{m}$. (c) Optical image of the 1L, ML, and 1L/ML devices fabricated from a single flake.

1L/ML interface, band bending induces a high local built-in electric field which can dissociate excitons efficiently [19] and enhance local photoresponse. With our contact geometry, the built-in field is perpendicular to the current channel, and cannot drive the dissociated free carriers to the contacts. The magnitude of photoresponse is expected to be lower than that with source-drain contacts on opposite sides of the interface. A source-drain bias is required to produce photocurrent in the circuit.

Fig. 2a shows the optical image of the scanned area $\left(20 \times 20 \mu \mathrm{m}^{2}\right)$. The widths of the $1 \mathrm{~L}$ and ML channels are $5 \mu \mathrm{m}$ and $4 \mu \mathrm{m}$, respectively. A bias voltage of $0.2 \mathrm{~V}$ was applied between the $V_{+}$and $V_{-}$contacts. The photocurrent maps were acquired with excitation wavelengths $660 \mathrm{~nm}(h \nu=1.88 \mathrm{eV}$, Fig. 2b), $680 \mathrm{~nm}(h \nu=1.83 \mathrm{eV}$, Fig. 2d), and $700 \mathrm{~nm}(h \nu=1.77 \mathrm{eV}$, Fig. 2f). A back-gate voltage of $20 \mathrm{~V}$ was applied during the scan. In our experiment, the intensity of the reflected laser and the photocurrent $\left(I_{\mathrm{pc}}\right)$ generated in the device were simultaneously recorded at each position, allowing the spatial photocurrent map to be correlated with the device geometry. The inner edges of the $V_{+}$and $V_{-}$contacts and the 1L/ML boundary in Fig. 2a are marked by black solid lines and arrows in Fig. $2 \mathrm{~b}$. The highest $I_{\mathrm{pc}}$ intensity is observed at the inner edge of the $V_{+}$contact, which can be attributed to the local electrical field at the $\mathrm{Au} / \mathrm{MoS}_{2}$ interface and the bias voltage applied between the $V_{+}$and $V_{-}$contacts [18. To visualize the spatial dependence of $I_{\mathrm{pc}}$ in other areas, $I_{\mathrm{pc}}$ is plotted with a $\log$ scale in Fig. $2 \mathrm{~b}, 2 \mathrm{~d}$, and $2 \mathrm{f}$. The photocurrent line profiles at various wavelengths were also recorded across the $1 \mathrm{~L} / \mathrm{ML}$ boundary, shown in Fig. 2c, $2 \mathrm{e}$, and $2 \mathrm{~g}$. To avoid the influence of the high-intensity photocurrent at the $V_{+}$contact, the 

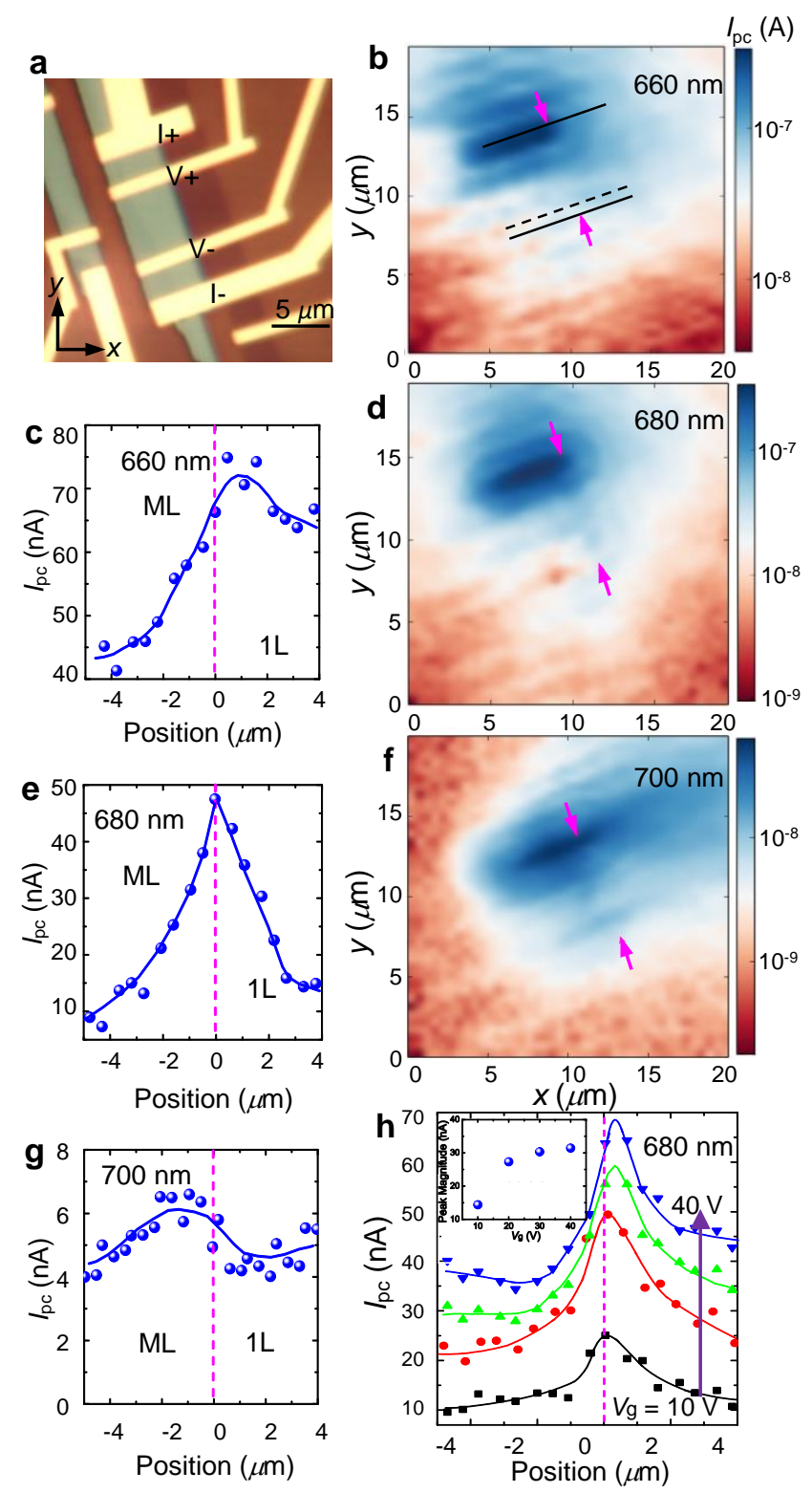

FIG. 2. Photocurrent generation in 1L/ML homostructures at various excitation wavelengths. (a) Optical image of the 1L+ML device. A bias voltage is applied between the $V_{+}$and $V_{-}$contacts. (b-f) Photocurrent intensity spatial maps with line profiles along the dashed line in $\mathrm{b}$ at excitation wavelength of $660 \mathrm{~nm}(\mathrm{~b}-\mathrm{c}), 680 \mathrm{~nm}$ (d-e), and $700 \mathrm{~nm}(\mathrm{f}-\mathrm{g}) . I_{\mathrm{pc}}$ is plotted on a log scale in spatial maps and with normal scale in line profiles. The black solid lines in b indicate inner edges of the $V_{+}$and $V_{-}$contacts. The pink arrows mark the $1 \mathrm{~L} / \mathrm{ML}$ boundary. In c, e, and g, the pink dashed lines separate the $1 \mathrm{~L}$ and $\mathrm{ML}$ regions. The solid lines are guides for eyes. The log scale reduces the prominence of the contact region, allowing the edge signal to be seen in the map, although the edge features are clearest in the line profiles. (h) Profiles of photocurrent along a fixed scan line perpendicular to the interface at $V_{\mathrm{g}}=10,20,30$, and $40 \mathrm{~V}$. The wavelength of the excitation laser is $680 \mathrm{~nm}$. Successive curves are offset by $7 \mathrm{nA}$ for better visualization. The inset shows the peak magnitude as a function of the gate voltage. 
line is chosen close to the $V_{-}$contact, as shown by the black dashed line in Fig. 2b.

Under illumination of $\lambda=680 \mathrm{~nm}$, a region with enhanced photocurrent is observed between the two arrows in Fig. 2d, overlapping with the 1L/ML boundary. A $I_{\mathrm{pc}}$ peak located at the boundary is also observed in the line profile in Fig. 2c. This confirms that the bent bands at the $1 \mathrm{~L} / \mathrm{ML}$ interface can increase local photocurrent generation. The enhanced photocurrent at the $1 \mathrm{~L} / \mathrm{ML}$ boundary is observed for various gating voltages $V_{\mathrm{g}}$, ranging from $10 \mathrm{~V}$ to $40 \mathrm{~V}$ (Fig. $2 \mathrm{~h}$ ). The $V_{\mathrm{g}}$ dependence of peak magnitude is much weaker than that observed on $\mathrm{MoS}_{2} /$ metal boundaries [20] because the global shifting of the back gate couldn't change the built-in potential at the $1 \mathrm{~L} / \mathrm{ML}$ interface. This observation is consistent with the $V_{\mathrm{g}}$-dependent $I_{\mathrm{pc}}$ with contacts on opposite sides of the junction [2, 12]. At $\lambda=660 \mathrm{~nm}$, the photon energy very nearly matches the optical band gaps in $1 \mathrm{~L}$ and ML $\mathrm{MoS}_{2}$. Photocurrent arising from other effects such as hot carriers [21] and photothermoelectric effect [22] increases in the interior of 1L and ML [15, 23] and reduces the intensity contrast at the $1 \mathrm{~L} / \mathrm{ML}$ boundary. At $\lambda=700 \mathrm{~nm}$, the photon energy is too low to effectively create excitons in $\mathrm{MoS}_{2}$. The overall photocurrent intensity is strongly reduced and no enhancement can be observed at the $1 \mathrm{~L} / \mathrm{ML}$ boundary.

Here, the photocurrent enhancement at the 1L/ML boundary is interpreted as arising from bent bands, but the possibility of a new state with optical band gap $1.83 \mathrm{eV}$ cannot be ruled out. Unusual edge states of TMDCs have been observed with a band gap smaller than that of the interior [24, 25]. An optically active band gap of $1.42 \mathrm{eV}$ was theoretically predicted and experimentally observed in $\mathrm{MoS}_{2} / \mathrm{WS}_{2}$ bilayer vertical heterostructures [2628]. Nevertheless, the enhanced photocurrent suggests that the $1 \mathrm{~L} / \mathrm{ML}$ interface exhibits new properties associated with the unique band structure of this boundary.

To probe the conducting properties of the interface charge state, we measured transport of devices with current flowing along a $1 \mathrm{~L}$ and ML boundary (1L+ML devices). The conductance of multiple composite 1L+ML devices were measured and compared with the independent $1 \mathrm{~L}$ and $\mathrm{ML}$ devices fabricated from the same sample. For each set of the $1 \mathrm{~L}$, $\mathrm{ML}$, and $1 \mathrm{~L}+\mathrm{ML}$ devices, the devices originate from the same single-crystalline flake and were processed with the same procedures. It can thus be assumed that the conductivity of the $1 \mathrm{~L}$ (or ML) device equals to that of the $1 \mathrm{~L}$ (or ML) channel in the $1 \mathrm{~L}+\mathrm{ML}$ device. This control design allows us to compare the effective conductivity of the $1 \mathrm{~L}+\mathrm{ML}$ device $\sigma_{\mathrm{eff}}=G \cdot \frac{L}{W_{\mathrm{m}}+W_{1}}=\frac{I_{\mathrm{sd}}}{V_{\mathrm{sd}}} \cdot \frac{L}{W_{\mathrm{m}}+W_{1}}\left(G\right.$ is the net conductance of the $1 \mathrm{~L}+\mathrm{ML}$ device, $W_{\mathrm{m}}, W_{1}$, and $L$ are the widths and length of the ML and $1 \mathrm{~L}$ channels in the $1 \mathrm{~L}+\mathrm{ML}$ device) with the conductivity of the independently measured $1 \mathrm{~L}$ and the ML devices, $\sigma_{1}$ and $\sigma_{\mathrm{m}}$, respectively. Typically $\sigma_{\mathrm{m}}$ is higher than $\sigma_{1}$ [29]. If the conductivity of the interface conduction channel is close to $\sigma_{1}$ or $\sigma_{\mathrm{m}}$, a naive analysis $\sigma_{\mathrm{m}}>\sigma_{\text {eff }}>\sigma_{1}$ should be observed. However, in all our measured devices, it is found that $\sigma_{\text {eff }}>\sigma_{\mathrm{m}}>\sigma_{1}$, suggesting an enhanced conductivity at the 1L/ML interface. Two examples are shown in Fig. 3.

Fig. 3a shows the room-temperature gate-dependent conduction properties of the $1 \mathrm{~L}$, $\mathrm{ML}$, and $1 \mathrm{~L}+\mathrm{ML}$ devices from 4-point measurement in Fig. 1c. Linear $I_{\mathrm{sd}}-V_{\mathrm{sd}}$ curves are observed in all the devices confirming Ohmic contacts (the inset of Fig. 3a and Fig. S3 in the Supplementary Information). The gating characteristics are plotted as an effective conductivity $G \frac{L}{W}$. The effective conductivity $\sigma_{\text {eff }}$ defined in this manner for the composite $1 \mathrm{~L}+\mathrm{ML}$ device represents the conductivity assuming the entire device was uniform; it is not the actual conductivity of any conduction channel in the device. The effective conductivity 
$G \frac{L}{W}$ is equivalent to the actual conductivity $\sigma_{1}$ and $\sigma_{\mathrm{m}}$ for the $1 \mathrm{~L}$ and the ML devices, respectively. Of the three devices, the $1 \mathrm{~L}+\mathrm{ML}$ has the highest effective conductivity over the full range of $V_{\mathrm{g}}$, indicating that the interface is more conductive than the $1 \mathrm{~L}$ or $\mathrm{ML}$ regions. The effective field-effect mobility of the $1 \mathrm{~L}+\mathrm{ML}$ device can be calculated using $\mu_{\mathrm{FE}-\mathrm{eff}}=\frac{1}{C_{\mathrm{i}}} \frac{\partial \sigma_{\mathrm{eff}}}{\partial V_{\mathrm{g}}}$ and compared with the field-effect mobilities of the $1 \mathrm{~L}$ and $\mathrm{ML}$ devices, $\mu_{\mathrm{FE}-1}=\frac{1}{C_{\mathrm{i}}} \frac{\partial \sigma_{1}}{\partial V_{\mathrm{g}}}$ and $\mu_{\mathrm{FE}-\mathrm{m}}=\frac{1}{C_{\mathrm{i}}} \frac{\partial \sigma_{\mathrm{m}}}{\partial V_{\mathrm{g}}}$, where $C_{\mathrm{i}}=1.3 \times 10^{-4} \mathrm{~F} / \mathrm{m}^{2}$ is the capacitance per unit area of our 285-nm-thick $\mathrm{SiO}_{2}$ layer [30]. The effective mobilities of these devices are $\mu_{\mathrm{FE}-1}=4.1 \mathrm{~cm}^{2} / \mathrm{Vs}, \mu_{\mathrm{FE}-\mathrm{m}}=11.4 \mathrm{~cm}^{2} / \mathrm{Vs}$, and $\mu_{\mathrm{FE}-\text { eff }}=20.2 \mathrm{~cm}^{2} / \mathrm{Vs}$. The value of $\mu_{\mathrm{FE}-1}$ is within the range of $0.1-10 \mathrm{~cm}^{2} / \mathrm{Vs}$ expected for uncapped, back-gated monolayer $\mathrm{MoS}_{2}$ FETs on $\mathrm{SiO}_{2} / \mathrm{Si}$ substrates. The increased conductivity and mobility of $\mathrm{ML} \mathrm{MoS}_{2}$ relative to the $1 \mathrm{~L}$ device is consistent with previous reports [29]. As with the effective conductivity $\sigma_{\text {eff }}$, the effective field-effect mobility $\mu_{\mathrm{FE}-\text { eff }}$ of the $1 \mathrm{~L}+\mathrm{ML}$ device is higher than that of the $1 \mathrm{~L}$ and $\mathrm{ML}$ channel, which further confirms that the $1 \mathrm{~L} / \mathrm{ML}$ interface has a significant impact on the conduction properties of the composite device.

The observed relative ordering of $\sigma_{\text {eff }}>\sigma_{\mathrm{m}}>\sigma_{1}$ and $\mu_{\mathrm{FE}-\mathrm{eff}}>\mu_{\mathrm{FE}-\mathrm{m}}>\mu_{\mathrm{FE}-1}$ is reproduced in additional $1 \mathrm{~L}+\mathrm{ML}$ devices. Fig. 3b compares the conductivity (or effective conductivity) of a $1 \mathrm{~L}$, a $2 \mathrm{~L}$, and a $1 \mathrm{~L}+2 \mathrm{~L}$ two-terminal FETs again fabricated from a same $\mathrm{MoS}_{2}$ flake. It is clearly observed that the effective conductivity of the $1 \mathrm{~L}+2 \mathrm{~L}$ device is higher than the conductivity of the $1 \mathrm{~L}$ and the $2 \mathrm{~L}$ devices. The field-effect mobility (or effective mobility) values are $\mu_{\mathrm{FE}-1}=2.8 \mathrm{~cm}^{2} / \mathrm{Vs}, \mu_{\mathrm{FE}-\mathrm{m}}=11.0 \mathrm{~cm}^{2} / \mathrm{Vs}$, and $\mu_{\mathrm{FE}-\mathrm{eff}}=15.2 \mathrm{~cm}^{2} / \mathrm{Vs}$. Transport properties of an additional 1L+ML device, temperature dependence, and a summary of all measured devices are presented in the Supplementary Information. As shown in the Supplementary, the enhanced effective conductivity persists at low temperatures and with different gate voltages. In every measured 1L+ML device, the presence of the $1 \mathrm{~L} / \mathrm{ML}$ interface enhances the effective conductivity over that of the independent $1 \mathrm{~L}$ and ML channels.

\section{Discussion}

The observations suggest a modified charge state at the 1L/ML interface that impacts longitudinal effective conductivity in the TMDC devices. Although the measurements clearly demonstrate increased effective conductivity and effective field-effect mobility for the composite 1L+ML devices, there are several possible interpretations of the underlying mechanism which are not yet clearly distinguished. One attractive explanation is the band bending charge accumulation model as depicted in Fig. 4. When the $1 \mathrm{~L}$ and $\mathrm{ML} \mathrm{MoS}_{2}$ are in contact, electrons from the $1 \mathrm{~L}$ region diffuse into the ML region because of the potential difference [31, resulting in bent bands near the interface, in analogy to the 2DEG in a $\mathrm{Al}_{x} \mathrm{Ga}_{1-x} \mathrm{As} / \mathrm{GaAs}$ heterojunction [4. The accumulated electrons are localized at the interface and perhaps experience reduced scattering in this localized state with increased carrier density, thereby promoting conductance in the device. Such a band diagram was also suggested by finite element device simulation [12] and Kelvin probe force microscopy [2]. The detailed band diagram of a 1L+ML interface can be more complex than the traditional band bending depicted in Fig. 4; it has been proposed that the edge state of the ML MoS ${ }_{2}$ can disrupt the band alignment because of its proximity to the 1L/ML interface [13]. Neverthe- 

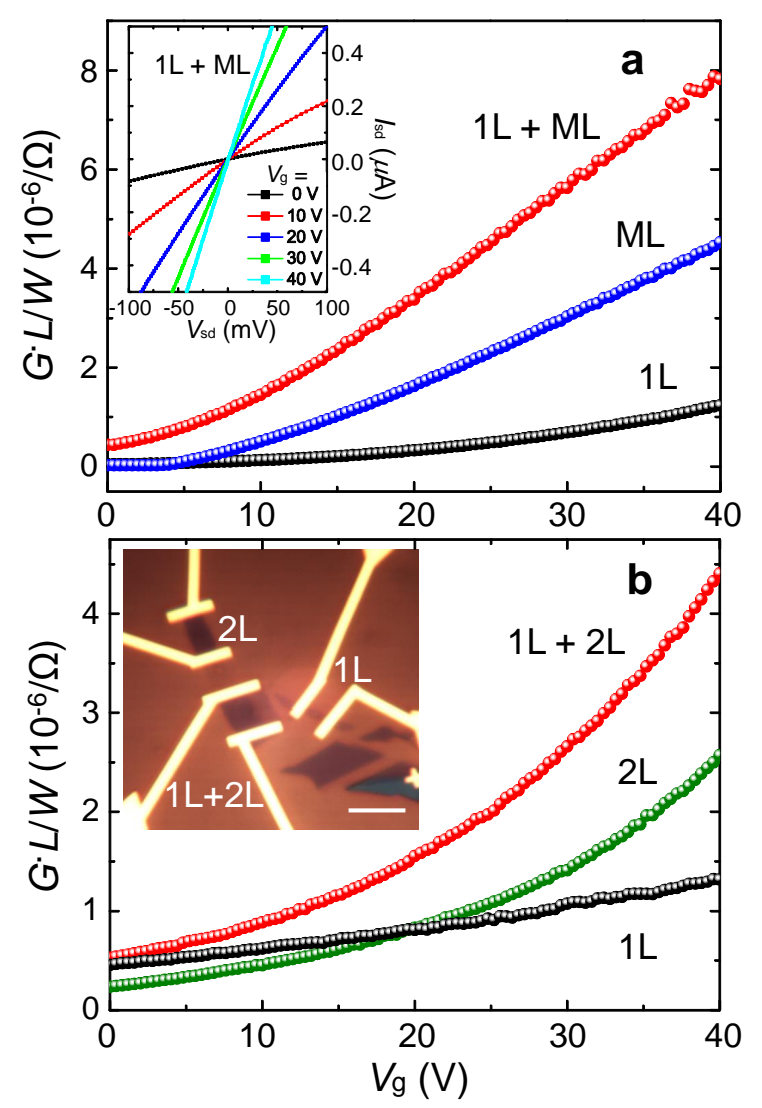

FIG. 3. Electronic properties of back-gated 1L+ML devices. (a) Effective conductivity $G L / W$ as a function of back-gate voltage for the $1 \mathrm{~L}, \mathrm{ML}$, and $1 \mathrm{~L}+\mathrm{ML}$ devices in Fig. 1c. The inset shows $I_{\mathrm{sd}}-V_{\mathrm{sd}}$ characteristics of the $1 \mathrm{~L}+\mathrm{ML}$ device at various gate voltages. (b) The gate-voltagedependent conductivity of the two-terminal $1 \mathrm{~L}, 2 \mathrm{~L}$, and $1 \mathrm{~L}+2 \mathrm{~L}$ devices shown in the inset. The scale bar is $5 \mu \mathrm{m}$.

less, this model suggests a mechanism based on established semiconductor heterostructure physics for the accumulation of charge at the interface.

Another possible explanation is the proposed existence of a metallic edge state at the boundary of $2 \mathrm{D} \mathrm{MoS}$ flakes [6, 10, 11, 33]. Considering that the edges of 1L and ML devices are also contacted with Au contacts, any such metallic edge state conductance should also contribute to $\sigma_{1}$ and $\sigma_{\mathrm{m}}$. The conductivity contribution from the edge state, which can be roughly compared using the number of edges divided by the width, should be smaller in $1 \mathrm{~L}+\mathrm{ML}$ device $(\sim 3 / 9 \mu \mathrm{m}$ for the device in Fig. 1c) and larger in $1 \mathrm{~L}(\sim 2 / 3 \mu \mathrm{m}$, Fig. 1c) or ML $(\sim 2 / 2.5 \mu \mathrm{m}$, Fig. 1c) device. There is still no direct transport report proving the metallic conductivity at the independent edge of $1 \mathrm{~L}$ or $\mathrm{ML} \mathrm{MoS}_{2}$, and the enhanced conduction that we observe are specific to the $1 \mathrm{~L} / \mathrm{ML}$ interface, not $\mathrm{MoS}_{2}$ edge. Thus the edge state of the 2D material is not likely to be solely responsible for the enhanced effective conductivity and mobility in our measurements.

More relevant here, conductive edge states at $\mathrm{MoS}_{2}$ layer dislocations have also been measured using microwave impedance microscopy [14]. Our results of interface band bending and 

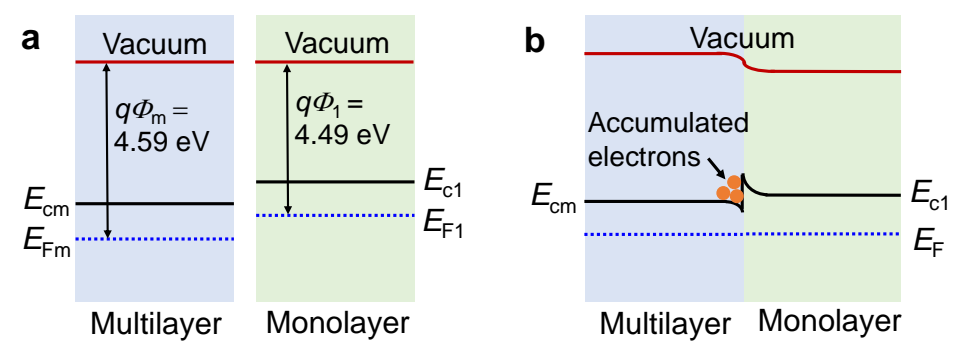

FIG. 4. Schematic band diagrams. (a) Schematic band diagrams of a multilayer and a monolayer $\mathrm{MoS}_{2} . q \Phi_{1}$ and $q \Phi_{\mathrm{m}}$ are the work functions of $1 \mathrm{~L}$ and $\mathrm{ML} \mathrm{MoS}_{2}$, respectively [31. (b) Schematic band diagram of $\mathrm{MoS}_{2} 1 \mathrm{~L} / \mathrm{ML}$ homojunction in thermal equilibrium.

enhanced longitudinal conduction support this picture of a narrow confined low-dimensional conducting charge accumulation, perhaps induced by the band bending known to occur at the 1L/ML interface from the photocurrent measurements. With now several reports of conducting boundaries between $\mathrm{MoS}_{2}$ regions of different layer number, additional theoretical and experimental investigation is required to construct a clear interpretation of the origin of these interfacial electronic states and their enhanced conductivity.

Despite the open question of the precise microscopic origin of the enhanced effective conductivity at $1 \mathrm{~L} / \mathrm{ML}$ interfaces, we use a simple electron affinity model to interpret the transport results based on the success of similar band engineering of interface states in $2 \mathrm{D}$ interfaces of 3D heterostructures [3]. A heterojunction band model is applied to explore the properties of the confined interfacial edge states (See the Supplementary Information). The width of depletion region $x_{1}$, the width of accumulation region $x_{\mathrm{m}}$ and the carrier density at the interface $N_{\text {di }}$ can be extracted from the model. The values for the $1 \mathrm{~L}+\mathrm{ML}$ device shown in Fig. $1 \mathrm{c}$ are $x_{1}=1.2 \mathrm{~nm}, x_{\mathrm{m}}=2.3 \mathrm{~nm}$, and $N_{\mathrm{di}}=7.7 \times 10^{12} \mathrm{~cm}^{-2}$. Because of the weaker electron screening in a $2 \mathrm{D}$ material with respect to that in a $3 \mathrm{D}$ material, the interface electrons can spread over a wider range [32]. Our calculation may underestimate the $x_{\mathrm{m}}$ and $x_{1}$ values. Nevertheless, the value of the depletion width $x_{1}$ is in reasonable agreement with the band profile at the $\mathrm{MoS}_{2}$ /graphene interface (depletion width $\sim 5 \mathrm{~nm}$ ) [33] directly imaged by scanning tunneling microscopy. $N_{\mathrm{di}}$ is about 10 times $N_{\mathrm{dm}}$ and $N_{\mathrm{d} 1}$, suggesting electron accumulation at the interface. Considering that the interface electrons are spatially separated from impurities, a significant scattering source in the interior, they may possess higher mobility than that of interior electrons. Based on the band bending and metallic edge state evidence here and elsewhere [13, 14, a highly conductive electronic accumulation is a reasonable explanation for the enhanced longitudinal conduction at $1 \mathrm{~L} / \mathrm{ML}$ interfaces compared to separate 1L and ML devices.

\section{Conclusion}

In summary, we have fabricated and investigated $\mathrm{MoS}_{2}$ monolayer/multilayer homojunctions with source-drain bias applied longitudinally along the interface. Scanning photocurrent microscopy reveals enhanced photoresponse at the $1 \mathrm{~L} / \mathrm{ML}$ interface, which can be explained by the band bending at the interface. Electronic transport measurements of homojunctions compared to distinct $1 \mathrm{~L}$ and ML devices provide evidence of enhanced con- 
ductivity along the boundary, indicative of a modified interfacial charge state. Although the precise microscopic mechanism driving this enhanced conduction is still not clear, our measurements reveal that the TMDC homojuction interface has a non-trivial impact on longitudinal conductivity of a composite layered device. Further exploration and exploitation of these band engineering edge features in layered heterojunction devices can open a potential pathway to achieve a confined metallic 1D electronic state in TMDCs [34].

\section{Acknowledgments}

The work was supported by the Institute for Sustainability and Energy at Northwestern (ISEN) and by the U.S. Department of Energy, Office of Basic Energy Sciences under award number de-sc0012130 (scanning photocurrent). Preparation of layered samples for this work was partially supported by the National Science Foundations MRSEC program (DMR- 1121262) and made use of its Shared Facilities at the Materials Research Center of Northwestern University. Characterization made use of the NIFTI facility in Northwestern University's Atomic and Nanoscale Characterization Experimental Center (NUANCE), which has received support from the MRSEC program (NSF DMR-1121262) at the Materials Research Center, the Nanoscale Science and Engineering Center (NSF EEC-0118025/003), the State of Illinois, and Northwestern University. Portions of device fabrication were performed at Northwestern University Micro/Nano Fabrication Facility (NUFAB), which is partially supported by Soft and Hybrid Nanotechnology Experimental (SHyNE) Resource (NSF NNCI-1542205), the MRSEC program (NSF DMR-1121262), the State of Illinois, and Northwestern University. The authors acknowledge V. Chandrasekhar for assistance with facilities and instrumentation. N.P.S. acknowledges support as a Research Fellow of the Alfred P. Sloan Foundation.

[1] T. Cheiwchanchamnangij and W. R. L. Lambrecht. "Quasiparticle band structure calculation of monolayer, bilayer, and bulk $\mathrm{MoS}_{2} . "$ Phys. Rev. B 85, 205302 (2012).

[2] M. Tosun, D. Fu, S. B. Desai, C. Ko, J. S. Kang, D.-H. Lien, M. Najmzadeh, S. Tongay, J. Wu, and A. Javey. " $\mathrm{MoS}_{2}$ heterojunctions by thickness modulation." Sci. Rep. 5, 10990 (2015).

[3] S. M. Sze. Semiconductor Devices: Physics and Technology Ch. 4 (John Wiley and Sons, Inc, 2001).

[4] T. Ando, A. B. Fowler. and F. Stern. "Electronic properties of two-dimensional systems." Rev. Mod. Phys. 54, 437 (1982).

[5] Q. L. He, H. Liu, M. M. He, Y. H. Lai, H. He, G. Wang, K. T. Law, J. Wang, and I. K. Sou. "Two-dimensional superconductivity at the interface of a $\mathrm{Bi}_{2} \mathrm{Te}_{3} / \mathrm{FeTe}$ heterostructure." Nat. Comm. 5, 4247 (2004).

[6] M. V. Bolllinger, J. V. Lauritsen, K. W. Jacobsen, J. K. Norskov, S. Helveg, and F. Besenbacher. "One-dimensional metallic edge states in $\mathrm{MoS}_{2}$." Phy. Rev. Lett. 87, 196903 (2001).

[7] A. Vojvodic, B. Hinnemann, and J. K. Norskov. "Magnetic edge states in $\mathrm{MoS}_{2}$ characterized using density-functional theory." Phys. Rev. B 80, 125416 (2009). 
[8] W. Bao, et al. "Visualizing nanoscale excitonic relaxation properties of disordered edges and grain boundaries in monolayer disulfide." Nat. Comm., 6, 7993 (2015).

[9] Y. L. Huang, et al. "Bandgap tunability at single-layer molybdenum disulphide grain boundaries." Nat. Comm. 6, 6289 (2015).

[10] Y. Li, Z. Zhou, S. Zhang, Z. Chen. " $\mathrm{MoS}_{2}$ nanoribbons: High stability and unusual electronic and magnetic properties." J. Am. Chem. Soc. 130, 16739-16744 (2008).

[11] J. Xiao, et al. "Carrier mobility of $\mathrm{MoS}_{2}$ nanoribbons with edge chemical modification." Phys. Chem. Chem. Phys. 17, 68656873 (2015).

[12] S. L. Howell, D. Jariwala, C.-C. Wu, K.-S. Chen, V. K. Sangwan, j. Kang, T. J. Marks, M. C. Hersam and L. Lauhon. "Investigation of band-offsets at Monlayer-multilayer $\mathrm{MoS}_{2}$ junctions by scanning photocurrent microscopy ." Nano Lett. 15, 2278-2284 (2015).

[13] Y. Guo. et al. "Edge-state-induced disruption to the energy band alignment at thicknessmodulated molybdenum sulfide junctions ." Adv. Electron. Mater. 1600048 (2016).

[14] D. Wu, X. Li, L. Luan, X. Wu, W. Li, M. N. Yogeesh, R. Ghosh, Z. Chu, D. Akinwande, Q. $\mathrm{Niu}$, and K. Lai. "Uncovering edge states and electrical inhomogeneity in $\mathrm{MoS}_{2}$ field-effect transistors." Proc. Nat. Acad. Sci. 113, 8583-8588 (2016).

[15] K. F. Mak, C. Lee, J. Hone, J. Shan, and T .F. Heinz. "Atomically thin $\mathrm{MoS}_{2}$ : a new direct-gap semiconductor." Phys. Rev. Lett. 105, 136805 (2010).

[16] A. Castellanos-Gomez, M. Buscema, R. Molenaar, V. Singh, L. Janssen, H. S. J. van der Zant, and G. A. Steele. "Deterministic transfer of two-dimensional materials by all-dry viscoelastic stamping." 2D Mater. 1, 011002 (2014).

[17] F. K. Perkins, A. L. Friedman, E. Cobas, P. M. Campbell, G. G. Jernigan, and B. T. Jonker. "Chemical vapor sensing with monolayer $\mathrm{MoS}_{2}$." Nano Lett. 13, 668-673 (2013).

[18] Wu C-C, D. Jariwala, V. K. Sangwan, T. J. Marks, M. C. Hersam and L. J. Lauhon. "Elucidating the photoresponse of ultrathin $\mathrm{MoS}_{2}$ field-effect transistors by scanning photocurrent microscopy." J. Phys. Chem. Lett. 4, 2508-2513 (2013).

[19] A. R. Klots. et al. "Probing excitonic states in ultraclean suspended two-dimensional semiconductors by photocurrent spectroscopy." Sci. Rep. 4, 6608 (2014).

[20] Li H-M, Lee D-Y, Choi M S, Qu D, Liu X, Ra C-H, Yoo W J 2014 Metal-Semiconductor Barrier Modulation for High Photoresponse in Transition Metal Dichalcogenide Field Effect Transistors. Sci. Rep 4, 4041; DOI: 10.1038/srep04041.

[21] Gabor N M, Song J C W, Ma Q, Nair N L, Taychatanapat T, Watanabe K, Taniguchi T, Levitov L S and Jarillo-Herrero P 2011 Hot carrier assisted intrinsic photoresponse in graphene. Science 334, 648-652.

[22] M. Buscema, M. Barkelid, V. Zwiller, H. S. van der Zant, G. A. Steele, and A. CastellanosGomez. "Large and tunable photothermoelectric effect in single-layer $\mathrm{MoS}_{2}$." Nano Lett. 13, 358-363 (2013).

[23] Z. Yin, H. Li, L. Jiang, Y. Shi, Y. Sun, G. Lu, Q. Zhang, X. Chen and H. Zhang. "Single-layer $\mathrm{MoS}_{2}$ phototransistors." ACS Nano 6, 74-80 (2012).

[24] A. M. van der Zande, P. Y. Huang, D. A. Chenet, T. C. Berkelbach, y. You, G.-H. Lee, T. F. Heinz, D. R. Reichman, D. A. Muller, and J. C. Hone. "Grains and grain boundaries in highly crystalline monolayer molybdenum disulphide." Nature Mater. 12, 554-561 (2013).

[25] H. R. Gutierrez, N. Perea-Lopez, A. L. Elias, A. Berkdemir, B. Wang, R. Lv, F. LopezUrias, V. H. Crespi, H. Terrones, H and M. Terrones. "Extraordinary room-temperature 
photoluminescence in triangular $\mathrm{WS}_{2}$ Monolayers." Nano Lett. 13, 3447-3454 (2013).

[26] Y. Gong et al. "Vertical and in-plane heterostructures from $\mathrm{WS}_{2} / \mathrm{MoS}_{2}$ monolayers Nature Mater. 13, 1135-1142 (2014).

[27] K. Kośmider and J. Fernandez-Rossier. "Electronic properties of the $\mathrm{MoS}_{2}-\mathrm{WS}_{2}$ heterojunction." Phys. Rev. B 87, 075451 (2013).

[28] H. Terrones, F. Lopez-Urias, and M. Terrones. "Novel hetero-layered materials with tunable direct band gaps by sandwiching different metal disulfides and diselenides." Sci. Rep. 3, 1549 (2013).

[29] S. L. Li, K. Wakabayashi, Y. Xu, S. Nakaharai, K. Komatsu, W.-W. Li, Y.-F. Lin, A. Aparecido-Ferreira, K. Tsukagoshi. "Thickness-dependent interfacial coulomb scattering in atomically thin field-effect transistors." Nano Lett. 13, 3546-3552 (2013).

[30] B. Radisavljevic, A. Radenovic, J. Brivio, V. Giacometti, and A. Kis. "Single-layer $\mathrm{MoS}_{2}$ transistors." Nature Nanotech. 6, 147-150 (2011).

[31] O. Ochedowski, K. Marinov, N. Scheuschner, A. Poloczek, B. K. Bussmann, J. Maultzsch, and M. Schleberger. "Effect of contaminations and surface preparation on the work function of single layer $\mathrm{MoS}_{2}$." Beilsterin J. Nanotechnol. 5, 291-297 (2014).

[32] V. K. Gurugubelli and S. Karmalkar. "Analytical theory of the space-charge region of lateral p-n junctions in nanofilms." J. Appl. Phys. 118, 034503 (2015).

[33] C. Zhang, A. Johnson, C.-L. Hsu, L.J. Li, and C.-K. Shih. "Direct imaging of band profile in single layer $\mathrm{MoS}_{2}$ on graphite: quasiparticle energy gap, metallic edge states, and edge band bending." Nano Lett. 14, 2443-2447 (2014).

[34] A. Ron and Y. Dagan. "One-dimensional quantum wire formed at the boundary between two insulating $\mathrm{LaAlO}_{3} / \mathrm{SrTiO}_{3}$ interfaces". Phys. Rev. Lett. 112136801 (2014). 